\title{
Evaluation of Trastuzumab-induced early cardiac dysfunction using two-dimensional Strain Echocardiography.
}

\author{
Sadik Volkan Emren ${ }^{1}$, Selcen Yakar Tuluce ${ }^{2}$, Fatih Levent ${ }^{2}$, Kamil Tuluce ${ }^{3}$, Toygar Kalkan ${ }^{4}$, \\ Yasar Yildiz ${ }^{5}$, Ahmet Alacacioğlư ${ }^{5}$, Yüksel Kucukzeybek ${ }^{5}$, Murat Akyol ${ }^{5}$, Tarık Salman ${ }^{5}$
}

\begin{abstract}
${ }^{1}$ Afyonkarahisar State Hospital, Department of Cardiology, ${ }^{2}$ Katip Celebi University Ataturk Training and Research Hospital, Department of Cardiology, ${ }^{3}$ Tepecik Training and Research Hospital, Department of Cardiology, ${ }^{4}$ Katip Celebi University Ataturk Training and Research Hospital, Department of Internal Medicine, ${ }^{5}$ Katip Celebi University Ataturk Training and Research Hospital, Department of Medical Oncology, Izmir, Turkey
\end{abstract}

\begin{abstract}
Aim: Trastuzumab, a chemotherapeutic agent used in the treatment of breast cancer. has been shown to induce subclinical left ventricular (LV) dysfunction during a three to six month period as evidenced by strain echocardiographic examination without any change occurring in the ejection fraction of LV. The present study evaluated the presence of subclinical LV dysfunction using strain echocardiography 1 day and 7 days after the initiation of trastuzumab therapy. Material and methods: The patients with breast cancer receiving adjuvant trastuzumab therapy underwent 2-dimensional, tissue Doppler, and strain echocardiographic examination at baseline and 1 day and 7 days after therapy. LV global longitudinal strain (GLS), global circumferential strain (GCS) values, and other echocardiographic parameters were calculated. Results: A total of 40 females, mean age $50 \pm 10$ years, were evaluated. Of these patients, $97 \%$ received anthracycline and $73 \%$ received radiotherapy before the initiation of trastuzumab therapy. No change was observed in any of the echocardiographic parameters 1 day after the initiation of trastuzumab therapy ( $\mathrm{p}>0.05$ ). The LV ejection fraction, tissue Doppler parameters, and GCS values did not show any changes 7 days after the initiation of therapy, whereas significant decreases were observed in GLS value $(19.2 \pm 4.0 \%$ vs. $17.2 \pm 3.4, p=0.001)$ and systolic annular velocity of the lateral LV wall ( $S$ ' velocity) $(10.5 \pm 3.2$ vs. 8.6 \pm 2.2 , $p=0.002)$. Conclusion: Trastuzumab therapy is associated with subclinical LV dysfunction as early as 7 days after initiation of the therapy as evidenced by the decreases in GLS value of LV and systolic annular velocity of the lateral LV wall.
\end{abstract}

Keywords: strain echocardiography, trastuzumab, cardiotoxicity

\section{Introduction}

Breast cancer is the most common cancer among women worldwide [1]. Many treatment options are available for breast cancer including medical and surgical therapies. Among these options trastuzumab, a chemotherapeutic agent used as an adjuvant therapy in breast cancer, exerts its effects by inhibiting Erbb-2 receptors

Received 23.05.2015 Accepted 13.08.2015

Med Ultrason

2015, Vol. 17, No 4, 496-502

Corresponding author: Sadık Volkan Emren

Afyonkarahisar State Hospital,

Department of Cardiology

73 Orhangazi Street Nedim Helvacioglu Bd

03000 Afyonkarahisar, Turkey

Phone: +9005052644578

E-mail: vemren@hotmail.com that belong to the family of epidermal growth factors [2]. Erbb-2 is overexpressed in around 30\% of women with breast cancer, and it is regarded as an indicator of poor prognosis [2]. The combined use of trastuzumab with other chemotherapeutic agents such as anthracyclines decrease 1 -year mortality rate by $11 \%$ [3]. However, trastuzumab therapy is associated with cardiotoxicity, and this side effect is even more common when trastuzumab is used with anthracyclines that were also previously shown to have cardiotoxic effects [4].

In daily practice cadiotoxic side effects of trastuzum$\mathrm{ab}$ and other cardiotoxic chemotherapeutic agents are determined by the measurement of left ventricular ejection fraction (LVEF) using 2-dimensional transthoracic echocardiography (TTE). However, the measurement of LVEF allows detection of cardiotoxicity only in later stages [5]. Therefore, attempts have been made to find 
new imaging methods in order to detect cardiotoxic side effects at an earlier stage [6]. One of these methods is strain echocardiography that provides important information about myocardial functions [7]. It was shown using strain echocardiography that trastuzumab therapy produces subclinical LV dysfunction after 3 and 6 months of therapy, without modifying the ejection fraction [8]. It is, however, unknown whether subclinical LV dysfunction occurs at an earlier stage.

The main aim of our study was to evaluate the presence of subclinical LV dysfunction using strain echocardiography (STE) as early as 1 day and 7 days after the initiation of trastuzumab therapy.

\section{Material and methods}

The study was conducted in patients with breast cancer followed by the medical oncology and cardiology clinics of Izmir Katip Celebi University Ataturk Training and Research Hospital and who had received trastuzumab therapy, between January 2014 and March 2014. The patients provided written and oral informed consent for participation in the study and for the administration of trastuzumab therapy. Approval was obtained from the local Ethics Committee.

Human epidermal growth factor receptor 2 (HER 2) status was determined using fluorescent in situ hybridization (FISH) or immunohistochemical (IHC) analysis in paraffin-embedded tissue blocks. The HER2 test result was reported as 'positive' if it is ( $3+)$ intense staining was obtained in IHC examination or amplification of HER2 genes using FISH technique. FISH technique was routinely performed, if $2+$ staining score was observed in IHC. Trastuzumab therapy was administered according to the international guidelines for the treatment of breast cancer. Accordingly, trastuzumab $8 \mathrm{mg} / \mathrm{kg}$ loading dose was followed by a $6-\mathrm{mg} / \mathrm{kg}$-maintenance dose one every three weeks (in $250 \mathrm{ml} 0.9 \%$ sodium chloride solution as 30 min intravenous infusion) [9]. Patients with coronary artery disease or anginal symptoms, valvular disease, atrial fibrillation, LV systolic dysfunction ( $\mathrm{LVEF}<50 \%$ ), acute or chronic renal failure, poor imaging quality were not included and those receiving cardiotoxic drug therapies other than anthracyclines were excluded. Other causes which might lead to cardiac dysfunction electrolyte imbalance, anemia, thyroid disease, connective tissue disorders, and hematological disorders were not included. Age, gender, height, weight, body mass index (BMI), previous therapy with anthracyclines and doses of previous anthracycline regimens, tumor localization, history of hypertension (HT), diabetes mellitus (DM), smoking, tumor stage, and history of surgery and radiotherapy were recorded.

\section{Echocardiographic study}

All subjects underwent evaluation with TTE, tissue Doppler, and strain imaging at baseline and 1 day and 7 days after the initiation of trastuzumab therapy. A commercially available ultrasound machine (i.E33, Philips Medical Systems, Andover, Mass) equipped with an $\mathrm{S} 5$ probe (2 to $4 \mathrm{MHz}$ ) was used in all echocardiographic examinations. Two independent readers evaluated echocardiographic recordings of the patients. Standard 2D and Doppler echocardiography were performed according to the recommendations of American Society of Echocardiography/European Association of Echocardiography. Two independent and experienced echocardiographers, who were blinded to clinical characteristics of the patients and trastuzumab therapy status, performed and evaluated echocardiographic recordings. LVEF was measured using the biplane Simpson's method [10]. Mitral inflow velocities were studied using pulsed-wave (PW) Doppler after placing the sample volume at the leaflets' tips [11]. The peak early (E-wave) and late filling (A-wave) velocities were measured. The Right ventricular (RV) inflow velocities (E and A waves) were determined after placing the sample volume at the leaflets' tips. The sample volume of PW Doppler was placed at the lateral and septal sides of the mitral annulus and lateral tricuspid annulus to obtain tissue Doppler Imaging (TDI) velocities. Systolic annular velocity (s'), early (e') and late (a') diastolic annular velocities of two ventricles were measured.

\section{Strain Echocardiography}

Strain measurements were performed using custom software (MVQ, QLAB, Philips). Digital cineloops from the apical four-,two-, and three-chamber views and parasternal short axis views at the basal, midventricular, and apical levels were obtained at a frame rate of 50-90 frames/sec at end-expiration were acquired from the peak of the R-wave and stored in optical disks in the Digital Imaging and Communications in Medicine (DICOM) format for offline analysis. The averages of three cardiac cycles were used in the analysis. For each of the 3 short-axis views, the sampling points were placed manually along the endocardium at LV base, middle and apex, and for apical 2-, 3-, and 4-chamber views, 3 sampling points were placed manually at septal mitral annulus, lateral corner and apical endocardium during end-diastole. The software tracked endocardial contour automatically generating a region of interest. The quality of myocardial tracking was checked visually, and the process was repeated or manually corrected if unsatisfactory tracking was obtained. The graphics of deformation parameters of each segment were then automatically formed and the average peak strain values were obtained. The global lon- 


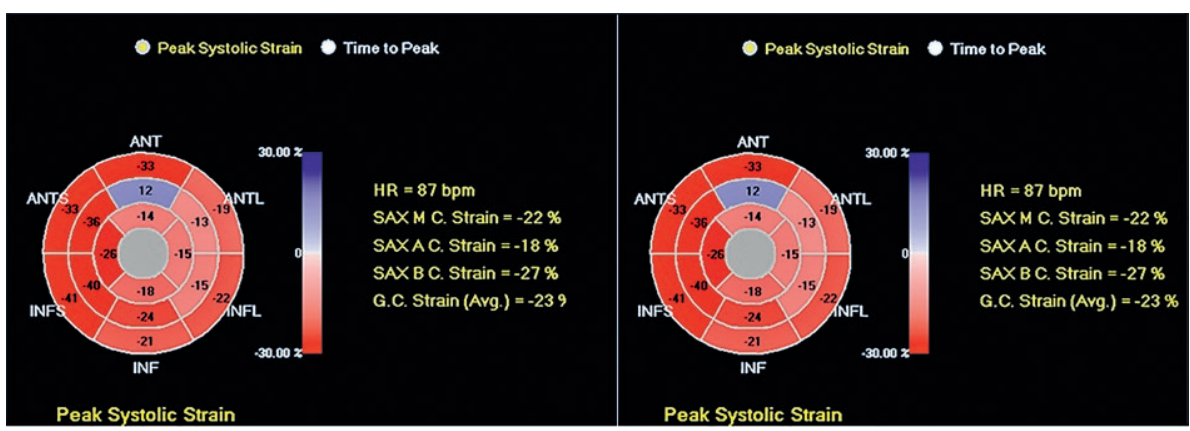

Fig 1. The image shows the global longitudinal and circumferential strain values which were assessed as the average of the segmental values

gitudinal strain (GLS) and global circumferential strain (GCS) values were assessed as the average of the segmental values (fig 1). According to the expert consensus for multimodality imaging evaluation of adult patients during and after cancer therapy, subclinical cardiac LV dysfunction was determined as a reduction in $>15 \%$ of basal GLS value of LV [12].

\section{Statistical analysis}

Statistical analysis was performed using SPSS software version 15 . The variables were investigated using histograms, probability plots, and analytical methods (Kolmogorov-Smirnov) to determine whether or not they were normally distributed. Descriptive statistics included mean and standard deviation (SD) for normally distributed variables and median and interquartile range (IQR) for not-normally distributed variables. The repeated measures analysis of variance test was used to evaluate changes in the parameters from baseline at days 1 and 7 after initiation of trastuzumab therapy. The numeric variables were compared between patients with or without subclinical dysfunction using the Student's t-test, and categoric variables were compared using chi-square test. Intraobserver and interobserver variability were evaluated in 10 random subjects by the intraclass correlation coefficient and by the coefficient of variation (CV) using the root-mean-square method. Overall, $5 \%$ type- 1 error level was used to infer statistical significance.

\section{Results}

The basic characteristics of the 40 patients enrolled are presented in Table I. Echocardiographic changes during trastuzumab therapy are presented in Table II. No changes were noted in LVEF during the course of the therapy. No changes were noted in tissue Doppler echocardiographic parameters of the RV. Mitral inflow parameters ( $\mathrm{E}$ and $\mathrm{A}$ waves) and GCS values were not signifi-
Table I. Baseline characteristics of the patients

\begin{tabular}{ll}
\hline Mean age (years) & $50 \pm 10$ \\
Height $(\mathrm{cm})$ & $158 \pm 6.3$ \\
Weight $(\mathrm{kg})$ & $75 \pm 15$ \\
BMI $(\mathrm{kg} / \mathrm{m} 2)$ & $30 \pm 1$ \\
Women & $40(100 \%)$ \\
Doxorubicin, n (\%) & $16(42 \%)$ \\
Epirubicin, n (\%) & $21(55 \%)$ \\
Doxorubicin + epirubicin, n (\%) & $1(3 \%)$ \\
Doxorubicin dose, mg/m2 & $431 \pm 79$ \\
Epirubicin dose, mg/m2 & $671 \pm 214$ \\
Stage & $3(8 \%)$ \\
$\quad$ Stage 1 & $17(43 \%)$ \\
$\quad$ Stage 2 & $7(18 \%)$ \\
$\quad$ Stage 3 & $13(33 \%)$ \\
Stage 4 & $20(50 \%)$ \\
Right breast, n (\%) & $18(45 \%)$ \\
Left breast, n (\%) & $2(5 \%)$ \\
Left and right breast, n (\%) & $36(95 \%)$ \\
Surgery, n (\%) & $29(73 \%)$ \\
Chest irradiation & $1(3 \%)$ \\
HT, n (\%) & $4(10 \%)$ \\
DM, n (\%) & $9(23 \%)$ \\
Smoking, n (\%) &
\end{tabular}

BMI, Body Mass Index; HT, Hypertension; DM, Diabetes Mellitus; Values are expressed as mean $\pm \mathrm{SD}$ or number (percentage).

cantly changed during the therapy. However, LV lateral s' velocity and GLS significantly decreased at 7 days after initiation of trastuzumab therapy. A similar change from baseline was not observed at day 1 .

From the entire population 25 patient developed trastuzumab mediated LV dysfunction. There was no difference between patients that developed cardiac dysfunction and those who did not develop cardiac dysfunction in terms of age, BMI, and clinical parameters (Table III). The patients that developed subclinical cardiac dysfunction had lower GLS values at day 7, while GCS and mitral inflow parameters (E and A waves) were similar (Table IV). 
Med Ultrason 2015; 17(4): 496-502

\begin{tabular}{|c|c|c|c|c|c|}
\hline & Pre-treatment & $\begin{array}{l}\text { Trastuzumab therapy } \\
\text { Day } 1\end{array}$ & $\begin{array}{l}\text { Trastuzumab therapy } \\
\text { Day } 7\end{array}$ & $\mathbf{p}^{*}$ & $p^{* * *}$ \\
\hline LVEF (\%) & $61 \pm 0.6$ & $60 \pm 0.6$ & $60 \pm 0.5$ & 0.09 & 0.35 \\
\hline GCS (\%) & $-21.6 \pm 4.2$ & $-21.1 \pm 4.9$ & $-21.3 \pm 3.6$ & 0.45 & 0.79 \\
\hline GLS (\%) & $-19.2 \pm 4.0$ & $-18.2 \pm 3.2$ & $-17.2 \pm 3.4$ & 0.05 & 0.001 \\
\hline $\mathrm{E}(\mathrm{cm} / \mathrm{s})$ & $83.1 \pm 10$ & $82.8 \pm 13$ & $84.7 \pm 10$ & 0.22 & 0.15 \\
\hline $\mathrm{A}(\mathrm{cm} / \mathrm{s})$ & $80.6 \pm 19$ & $81.0 \pm 22$ & $84.1 \pm 21$ & 0.18 & 0.40 \\
\hline $\mathrm{E} / \mathrm{A}$ & $1.1 \pm 0.4$ & $1.1 \pm 0.4$ & $1.1 \pm 0.3$ & 0.22 & 0.15 \\
\hline Ls' $(\mathrm{cm} / \mathrm{s})$ & $10.5 \pm 3.2$ & $10 \pm 2$ & $8.6 \pm 2.2$ & 0.11 & 0.002 \\
\hline Le' $(\mathrm{cm} / \mathrm{s})$ & $11.9 \pm 4$ & $12.1 \pm 3.1$ & $11.3 \pm 2.8$ & 0.16 & 0.82 \\
\hline $\mathrm{La}^{\prime}(\mathrm{cm} / \mathrm{s})$ & $11.7 \pm 3.7$ & $10.7 \pm 3.1$ & $10.7 \pm 3.2$ & 0.07 & 0.11 \\
\hline $\mathrm{Ss}^{\prime}(\mathrm{cm} / \mathrm{s})$ & $7.8 \pm 1.6$ & $7.5 \pm 2.1$ & $7.3 \pm 1.4$ & 0.22 & 0.59 \\
\hline $\mathrm{Se}^{\prime}(\mathrm{cm} / \mathrm{s})$ & $8.5 \pm 2.3$ & $9 \pm 2.4$ & $7.9 \pm 2.3$ & 0.66 & 0.19 \\
\hline $\mathrm{Sa}^{\prime}(\mathrm{cm} / \mathrm{s})$ & $9.6 \pm 2.2$ & $9.4 \pm 2.4$ & $9.2 \pm 3$ & 0.38 & 0.28 \\
\hline $\mathrm{Rs}^{\prime}(\mathrm{cm} / \mathrm{s})$ & $14.2 \pm 3.1$ & $13.9 \pm 2.4$ & $14.1 \pm 3.2$ & 0.22 & 0.45 \\
\hline $\mathrm{Re}^{\prime}(\mathrm{cm} / \mathrm{s})$ & $12.5 \pm 4.8$ & $12.1 \pm 4$ & $11.5 \pm 4.1$ & 0.33 & 0.15 \\
\hline $\mathrm{Ra}^{\prime}(\mathrm{cm} / \mathrm{s})$ & $14.9 \pm 4.7$ & $15.6 \pm 4.3$ & $13.9 \pm 4.6$ & 0.64 & 0.20 \\
\hline
\end{tabular}

A, left ventricular late diastolic inflow velocity; E, left ventricular early diastolic inflow velocity, LVEF, , left ventricular ejection fraction; GCS, Global Circumferential Strain; GLS, Global Longitudinal Strain; La', late diastolic annular velocity of the lateral left ventricular wall; Le', early diastolic annular velocity of the lateral left ventricular wall;Ls', systolic annular velocity of the lateral left ventricular wall; Ra', late diastolic annular velocity of the right ventricular wall; Re', early diastolic annular velocity of the right ventricular wall; Rs', systolic annular velocity of the right ventricular wall; Sa', late diastolic annular velocity of the septal ventricular wall; Se', early diastolic annular velocity of the septal ventricular wall; Ss', systolic annular velocity of the septal ventricular wall.

Values are expressed as mean $\pm \mathrm{SD}$ or number (percentage).

*: Denotes statistical difference between pre-treatment values and values recorded 1 day after the initiation of trastuzumab therapy

**: Denotes statistical difference between pre-treatment values and values recorded 7 days after the initiation of trastuzumab therapy

Table III. Baseline clinical parameters of patient with and without trastuzumab-mediated LV dysfunction.

\begin{tabular}{llll}
\hline & $\begin{array}{l}\text { Left ventri- } \\
\text { cle dysfunc- } \\
\text { tion (+) }\end{array}$ & $\begin{array}{l}\text { Left ventri- } \\
\text { cle dysfunc- } \\
\text { tion (-) }\end{array}$ & $\mathbf{p}$ \\
& $\mathbf{N}: 25$ & $\mathbf{N}: 15$ & \\
\hline Mean Age (years) & $48 \pm 2.2$ & $53 \pm 2.8$ & 0.129 \\
BMI (kg/m2) & $29 \pm 6.1$ & $32 \pm 6.8$ & 0.295 \\
Left side tumor & $14(\% 56)$ & $6(40 \%)$ & 0.327 \\
Anthracycline use & $21(84 \%)$ & $14(93 \%)$ & 0.633 \\
Late stage (3-4) & $12(48 \%)$ & $8(\% 53)$ & 0.744 \\
Chest irradiation & $16(64 \%)$ & $13(87 \%)$ & 0.120 \\
Smoking & $6(24 \%)$ & $3(20 \%)$ & 0.769 \\
DM & $1(4 \%)$ & $3(20 \%)$ & 0.139 \\
HT & $1(4 \%)$ & 0 & - \\
\hline
\end{tabular}

DM, Diabetes Mellitus; HT, Hypertension

Values are expressed as mean $\pm \mathrm{SD}$ or number (percentage).

The intraobserver intraclass coefficients for lateral s' velocity with TDI, GLS, and GCS were 0.95 (CV 2.5\%), 0.96 (CV 3.0\%), and 0.90 (CV 3.5\%), respectively. The interobserver intraclass coefficients for s' velocity with TDI, GLS, and GCS were 0.91 (CV 4.4\%), 0.90 (CV $5.4 \%$ ), and 0.80 (CV 5.7\%), respectively.

\section{Discussions}

The findings of the present study suggest that trastuzumab therapy can produce subclinical LV dysfunction as early as one week after initiation of trastuzumab therapy by resulting in reductions in GLS of LV and systolic tissue Doppler velocity of lateral LV wall.

In contrast to dose-dependent irreversible cardiotoxic effects associated with anthracyclines, trastuzumab therapy produces a dose-dependent and reversible cardiotoxicity $[13,14]$. It is considered that trastuzumab-related cardiotoxicity is caused by the inhibition of Erbb-2 receptors on the myocytes. The reason is that Erbb receptors play a role in myocyte viability and hypertrophy [15]. In experimental studies, inhibition of Erbb-2 and Erbb-4 receptors in mice was shown to cause cardiomyopathy in 8 to 12 weeks [16].

Apart from experimental methods and clinical research, cardiotoxic effects associated with trastuzumab therapy are examined in daily practice using calculation of LVEF. However, calculation of LVEF is not solely capable of detecting subclinical LV dysfunction [17]. Strain echocardiography is a new imaging method used to investigate the presence of subclinical LV dysfunction and provides important information about myocardial 
Table IV. Echocardiographic parameters of patients with and without Trastuzumab-mediated LV dysfunction.

\begin{tabular}{|c|c|c|c|}
\hline & $\begin{array}{l}\text { Left Ventricle } \\
\text { Dysfunction }(+) \\
\text { N:25 }\end{array}$ & $\begin{array}{l}\text { Left Ventricle } \\
\text { Dysfunction (-) } \\
\text { N:15 }\end{array}$ & p \\
\hline \multicolumn{4}{|l|}{ LVEF (\%) } \\
\hline Pretreatment & $61 \pm 5.4$ & $63 \pm 7.9$ & 0.222 \\
\hline Day 1 & $59 \pm 5.6$ & $62 \pm 7.4$ & 0.152 \\
\hline Day 7 & $60 \pm 5.2$ & $61 \pm 4.6$ & 0.442 \\
\hline \multicolumn{4}{|l|}{ GCS (\%) } \\
\hline Pretreatment & $22 \pm 4.5$ & $22 \pm 3.6$ & 0.851 \\
\hline Day 1 & $21 \pm 4.8$ & $22 \pm 5.6$ & 0.596 \\
\hline Day 7 & $17 \pm 3.7$ & $19 \pm 2.4$ & 0.713 \\
\hline \multicolumn{4}{|l|}{ GLS (\%) } \\
\hline Pretreatment & $18 \pm 2.1$ & $20 \pm 3.1$ & 0.065 \\
\hline Day 1 & $18 \pm 3.8$ & $19 \pm 1.9$ & 0.742 \\
\hline Day 7 & $17 \pm 3.7$ & $19 \pm 2.4$ & 0.045 \\
\hline \multicolumn{4}{|l|}{$\mathrm{Ls}^{\prime}(\mathrm{cm} / \mathrm{s})$} \\
\hline Pretreatment & $11 \pm 3.1$ & $11 \pm 3.5$ & 0.932 \\
\hline Day 1 & $10 \pm 1.6$ & $10 \pm 2.8$ & 0.510 \\
\hline Day 7 & $8 \pm 2$ & $9 \pm 2.7$ & 0.210 \\
\hline \multicolumn{4}{|l|}{$\mathrm{E}(\mathrm{cm} / \mathrm{s})$} \\
\hline Pretreatment & $83 \pm 11$ & $83 \pm 9$ & 0.939 \\
\hline Day 1 & $83 \pm 13$ & $82 \pm 13$ & 0.730 \\
\hline Day 7 & $85 \pm 11$ & $84 \pm 10$ & 0.909 \\
\hline \multicolumn{4}{|l|}{$\mathrm{A}(\mathrm{cm} / \mathrm{s})$} \\
\hline Pretreatment & $82 \pm 20$ & $79 \pm 19$ & 0.622 \\
\hline Day 1 & $81 \pm 25$ & $80 \pm 16$ & 0.888 \\
\hline Day 7 & $83 \pm 26$ & $86 \pm 8$ & 0.796 \\
\hline
\end{tabular}

A, left ventricular late diastolic inflow velocity; E, left ventricular early diastolic inflow velocity, LVEF, left ventricular ejection fraction; GCS, Global Circumferential Strain; GLS, Global Longitudinal Strain, Ls', systolic annular velocity of the lateral left ventricular wall

Values are expressed as mean $\pm \mathrm{SD}$ or number (percentage).

function. Strain is defined as the change in percentage or length from resting or original state. The deformation of myocardial segments indicates contraction and relaxation [18]. The studies on anthracyclines were the first to evaluate chemotherapeutic agent-induced LV dysfunction using strain echocardiography $[19,20]$. In subsequent years, studies on adjuvant trastuzumab therapy have been published.

Various studies have shown that adjuvant trastuzumab therapy can cause a decrease in Global radial strain, GCS, and GLS values without causing any change in LVEF in a three to six month period in patients with breast cancer [21-23]. Most of these studies suggested that GLS was a more valuable parameter for LV dysfunction and showed better correlation with cardiotoxicity in the long-term [24-26]. Age, characteristics and number of patients in the present study are comparable to many other studies. In addition, the rate of risk factors for coronary artery disease was also low, similar to other studies. However, a cardiotoxic agent, anthracycline therapy, was administered to almost all patients $(97 \%)$ in the current study and a high rate of patients $(73 \%)$ received radiotherapy. According to the above-mentioned studies, STE was able to detect adjuvant trastuzumab-associated subclinical LV dysfunction in the early period before any changes occurred in LVEF but STE was performed at the end of 3 months. Our study demonstrated that trastuzumab therapy could cause subclinical LV dysfunction by decreasing GLS values as early as one week after the initiation of the therapy.

Oxidative stress has a major role in mediating cardiotoxic effects of trastuzumab therapy. Increased production or insufficient elimination of reactive oxygen radicals due to inhibition of HER-2 receptors was shown to cause cardiomyocyte death [27]. Dirican et al reported increased level of reactive oxygen radicals and decreased level of antioxidant enzymes 1 day after the initiation of adjuvant trastuzumab therapy, and this finding was associated with the decrease in LVEF [28]. Based on this finding, increased level of free oxygen radicals after trastuzumab therapy was considered to cause impairment of longitudinal mechanical function of the left ventricle in the early period. The finding that trastuzumab therapy caused a decrease in GLS values in the early period and did not change GCS values was attributed to the longitudinal mechanical functions of left ventricle which is more vulnerable to myocardial diseases and stress [29]. In addition, patients that developed subclinical cardiac dysfunction had lower basal GLS values compared to patients that did not develop cardiac dysfunction and normal population, and therefore, longitudinal mechanical functions were already impaired in these patients [30]. Indeed, measurement of LVEF together with GLS is recommended to evaluate the presence of subclinical LV dysfunction associated with adjuvant trastuzumab therapy (8).

\section{Study limitations}

Although the present study suggests that trastuzumab therapy can induce subclinical LV dysfunction in the early period, it is not known whether the decrease of longitudinal strain is predictive of later cardiac events. Thus, longer periods of follow-up will be required. Also the number of patients who developed trastuzumab mediated LV dysfunction was small. Therefore, a larger population would be necessary to substantiate these findings.

\section{Conclusions}

The present study reported trastuzumab-induced subclinical LV dysfunction in the early period before any 
changes occurred in LVEF. Close monitoring of such patients in this early period may permit early initiation of cardioprotective measures before irreversible myocardial damage occurs.

\section{Conflict of interest: none}

\section{References}

1. Forouzanfar MH, Foreman KJ, Delossantos AM, et al. Breast and cervical cancer in 187 countries between 1980 and 2010: a systematic analysis. Lancet 2011; 378: 14611484.

2. Slamon DJ, Clark GM, Wong SG, Levin WJ, Ullrich A, McGuire WL. Human breast cancer: correlation of relapse and survival with amplification of the HER-2/neu oncogene. Science 1987; 235: 177-182.

3. Slamon DJ, Leyland-Jones B, Shak S, et al. Use of chemotherapy plus a monoclonal antibody against HER2 for metastatic breast cancer that overexpresses HER2. N Engl J Med 2001; 344: 783-792.

4. Seidman A, Hudis C, Pierri MK, et al. Cardiac dysfunction inthetrastuzumab clinical trials experience. J ClinOncol 2002; 20: 1215-1221.

5. Ewer MS, Ali MK, Mackay B, et al. A comparison of cardiac biopsy grades and ejection fraction estimations in patients receiving Adriamycin. J Clin Oncol 1984; 2: 112-117.

6. Kerkhove D, Fontaine C, Droogmans S, et al. How to monitor cardiac toxicity of chemotherapy: time is muscle! Heart 2014; 100: 1208-1217.

7. Marwick TH. Measurement of strain and strain rate by echocardiography: ready for prime time? J Am Coll Cardiol 2006; 47: 1313-1327.

8. Thavendiranathan P, Poulin F, Lim KD, Plana JC, Woo A, Marwick TH. Use of myocardial strain imaging by echocardiography for the early detection of cardiotoxicity in patients during and after cancer chemotherapy: a systematic review. J Am Coll Cardiol 2014; 63: 2751 2768.

9. Aebi S, Davidson T, Gruber G, Cardoso F; ESMO Guidelines Working Group. Primary breast cancer: ESMO Clinical Practice Guidelines for diagnosis, treatment and followup. Ann Oncol. 2011; 22: 12-24.

10. Lang RM, Bierig M, Devereux RB, et al. Recommendations for chamber quantification. Eur J Echocardiogr 2006; 7: 79-108.

11. Appleton CP, Jensen JL, Hatle LK, Oh JK. Doppler evaluation of left and right ventricular diastolic function: a technical guide for obtaining optimal flow velocity recordings. J Am Soc Echocardiogr 1997; 10: 271-292.

12. Plana JC, Galderisi M, Barac A, Ewer MS, et al. Expert consensus for multimodality imaging evaluation of adult patients during and after cancer therapy: a report from the American Society of Echocardiography and the European Association of Cardiovascular Imaging. J Am Soc Echocardiogr 2014; 27: 911-939.
13. Schwartz RG, McKenzie WB, Alexander J, et al. Congestive heart and left ventricular dysfunction complicating doxorubicin therapy. Am J Med 1987; 82: 1109-1118.

14. Ewer MS, Ewer SM. Cardiotoxicity of anticancer treatments: what the cardiologist needs to know. Nat Rev Cardiol 2010; 7: 564-575.

15. Zhao YY, Sawyer DR, Baliga RR, et al. Neuregulins promote survival and growth of cardiac myocytes: persistence of ErbB2 and ErbB4 expression in neonatal and adult ventricular myocytes. J Biol Chem 1998; 273: 10261-10269.

16. Ozcelik C, Erdmann B, Pilz B, et al. Conditional mutation of the ErbB2 (HER2) receptor in cardiomyocytes leads to dilated cardiomyopathy. Proc Natl Acad Sci U S A 2002; 99: 8880-8885.

17. Eidem BW. Identification of anthracycline cardiotoxicity: left ventricular ejection fraction is not enough. J Am Soc Echocardiogr 2008; 21: 1290-1292.

18. Marwick TH. Measurement of strain and strain rate by echocardiography: ready for prime time? J Am Coll Cardiol 2006; 47: 1313-1327.

19. Lipshultz SE, Alvarez JA, Scully RE. Anthracycline associated cardiotoxicity in survivors of childhood cancer. Heart 2008; 94: 525-533.

20. Altena R, Perik PJ, van Veldhuisen DJ, de Vries EG, Gietema JA. Cardiovascular toxicity caused by cancer treatment: strategies forearly detection. Lancet Oncol 2009; 10: 391-399.

21. Fallah-Rad N, Walker JR, Wassef A, et al. The utility of cardiac biomarkers, tissue velocity and strain imaging, and cardiac magnetic resonance imaging in predicting early left ventricular dysfunction in patients with human epidermal growth factor receptor II-positive breast cancer treated with adjuvant trastuzumab therapy. J Am Coll Cardiol 2011; 57: 2263-2270.

22. Sawaya H, Sebag IA, Plana JC, et al. Early detection and prediction of cardiotoxicity in chemotherapy-treated patients. Am J Cardiol 2011; 107: 1375-1380.

23. Sawaya H, Sebag IA, Plana JC, et al. Assessment of echocardiography and biomarkers for the extended prediction of cardiotoxicity in patients treated with anthracyclines, taxanes, and trastuzumab. Circ Cardiovasc Imaging 2012; 5: 596-603.

24. Negishi K, Negishi T, Hare JL, Haluska BA, Plana JC, Marwick TH. Independent and incremental value of deformation indices for prediction of trastuzumab-induced cardiotoxicity. J Am Soc Echocardiogr 2013; 26: 493-498.

25. Hare JL, Brown JK, Leano R, Jenkins C, Woodward N, Marwick TH. Use of myocardial deformation imaging to detect preclinical myocardial dysfunction before conventional measures in patients undergoing breast cancer treatment with trastuzumab. Am Heart J 2009; 158: 294-301.

26. Baratta S, Damiano M, Marchese M, et al. Serum markers, conventional Doppler echocardiography and two-dimensional systolic strain in the diagnosis of chemotherapy induced myocardial toxicity. Rev Argent Cardiol 2013; 81: 151-158.

27. Gordon LI, Burke MA, Singh AT, et al. Blockade of the erbB2 receptor induced cardiomyocyte death through mitochondrial and reactive oxygen species-dependent pathways. J Biol Chem 2009; 284: 2080-2087. 
28. Dirican A, Levent F, Alacacioglu A, et al. Acute cardiotoxic effects of adjuvant trastuzumab treatment and its relation to oxidative stress. Angiology 2014; 65: 944949.

29. Mor-Avi V, Lang RM, Badano LP, et al. Current and evolving echocardiographic techniques for the quantitative evaluation of cardiac mechanics: ASE/EAE consensus statement on methodology and indications endorsed by the Japanese Society of Echocardiography. J Am Soc Echocardiogr 2011; 24: 277-313.

30. Kocabay G, Muraru D, Peluso D, et al. Normal left ventricular mechanics by two-dimensional speckle-tracking echocardiography. Reference values in healthy adults. Rev Esp Cardiol 2014; 67: 651-658. 